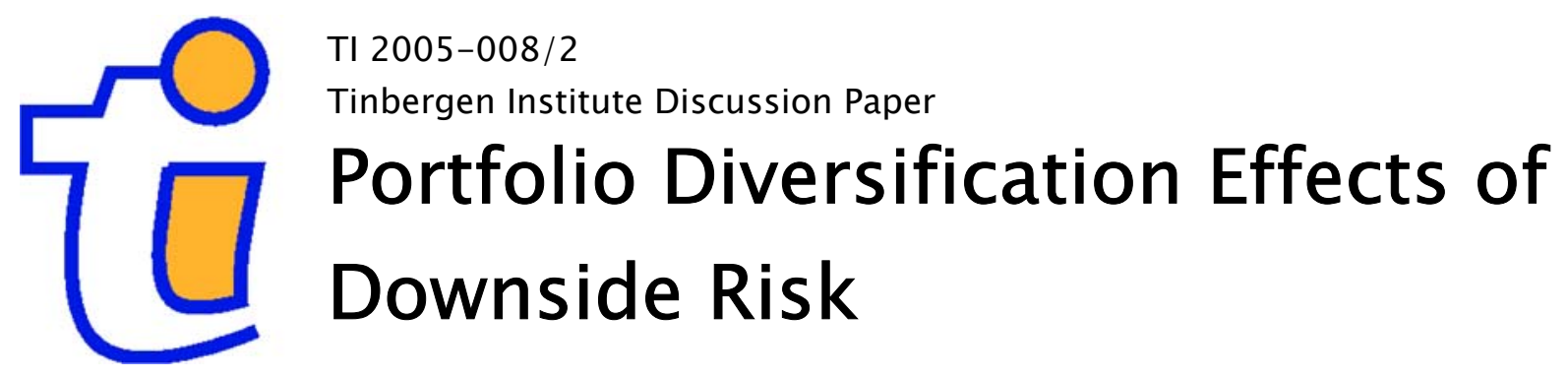

Namwon Hyung

Casper G. de Vries²

1 University of Seoul, South Korea,

2 Faculty of Economics, Erasmus University Rotterdam, and Tinbergen Institute. 
Tinbergen Institute

The Tinbergen Institute is the institute for economic research of the Erasmus Universiteit Rotterdam, Universiteit van Amsterdam, and Vrije Universiteit Amsterdam.

Tinbergen Institute Amsterdam

Roetersstraat 31

1018 WB Amsterdam

The Netherlands

Tel.: $\quad+31(0) 205513500$

Fax: $\quad+31(0) 205513555$

Tinbergen Institute Rotterdam

Burg. Oudlaan 50

3062 PA Amsterdam

The Netherlands

Tel.: $\quad+31(0) 104088900$

Fax: $\quad+31(0) 104089031$

Please send questions and/or remarks of nonscientific nature to driessen@tinbergen.nl.

Most TI discussion papers can be downloaded at http://www.tinbergen.nl. 


\title{
Portfolio Diversification Effects of Downside
}

\author{
Risk \\ Namwon Hyung and Casper G. de Vries \\ University of Seoul, Tinbergen Institute, and \\ Erasmus Universiteit Rotterdam
}

Febuary 2004, Revised October 2004

\begin{abstract}
Risk managers use portfolios to diversify away the un-priced risk of individual securities. In this paper we compare the benefits of portfolio diversification for downside risk in case returns are normally distributed with the case fat tailed distributed returns. The downside risk of a security is decomposed into a part which is attributable to the market risk, an idiosyncratic part and a second independent factor. We show that the fat-tailed based downside risk, measured as Value-at-Risk (VaR), should decline more rapidly than the normal based VaR. This result is confirmed empirically.
\end{abstract}

The first author benefitted from a research grant of the Tinbergen Institute. We are very grateful for the insightful comments of two referees, the editors and Thierry Post. Address correspondence to Casper G. de Vries, Department of Accounting and Finance H14-25, Erasmus University Rotterdam, PO Box 1738, 3000 DR, Rotterdam, The Netherlands, e-mail: cdevries@few.eur.nl (C. de Vries), nhyung@uos.ac.kr (N. Hyung) 
Keywords: Diversification, Value at Risk, Decomposition; JEL Clas-

sification: G0,G1,C2.

\section{Introduction}

Risk managers use portfolios to diversify away the un-priced risk of individual securities. This topic has been well studied for global risk measures like the variance, see e.g. the textbook by Elton and Gruber (1995, ch.4). In this paper we study the benefits of portfolio diversification with respect to extreme downside risk measure known as the zeroth lower partial moment and its inverse; where the inverse of the zeroth lower partial moment is better known as the VaR -Value at Risk- risk measure. Choice theoretic considerations for this risk measure are offered in Arzac and Bawa's (1977) analysis of the safety first criterion. In Gourieroux et al. (2000), the implications under the assumption of normally distributed returns are investigated, while Jansen et al. (2000) implement the safety first criterion for heavy tailed distributed returns. There is some concern in the literature that the VaR measure lacks subadditivity as a global risk measure. As a measure for the downside risk, however, the VaR exhibits subadditivity if one evaluates this criterion sufficiently deep in the tail area. ${ }^{1}$

The portfolio diversification effects for the downside risk are evaluated in terms of the diversification speed. The diversification speed is measured in two

\footnotetext{
${ }^{1}$ At least this holds for the normal distribution and the class of fat tailed distributions investigated in this paper.
} 
different ways. Let the "VaR-diversification-speed" be the rate at which the VaR changes as the number of assets $k$ included into the portfolio increases. Usually the safety first criterion and the VaR criterion are evaluated at a fixed probability level. It is also possible to do the converse analysis by fixing the VaR level and let the probability level change as the number of assets $k$ increases. This gives what we term the "Diversification-speed-of-the-risk-level". We will study both concepts. Much of the theoretical literature in finance presumes that the returns are normally distributed. For a host of questions this is a reasonable assumption to make. Empirically, it is well known that the return distributions have fatter tails than the normal, see e.g. Jansen and De Vries (1991). For the downside risk measures this data feature turns out to make a crucial difference. The diversification speeds are shown to be quite different for the cases of the normal and the fat tailed distributions. The VaR-diversification-speed is higher for the class of (finite variance) fat tailed distributions in comparison to the normal distribution, but is lower with respect to the Diversification-speed-ofthe-risk-level. The intuition for this result is as follows. Start with latter result. The tails of the normal density go down exponentially fast, while the tails of fat tailed distributions decline at a power rate (this is the defining characteristic of these distributions). Since an exponential function eventually beats any power, it stands to reason that the Diversification-speed-of-the-risk-level under normality is larger. The VaR-diversification-speed measures the speed in terms of quantiles, which are the inverse of the probabilities. Taking the inverse reverses the diversification speed. Consider for example the case of the normal 
versus Student-t distributed returns with $v$ degrees of freedom. It is well known that the VaR-diversification-speed for the normal distribution follows the square root rule. Per contrast, the Student-t VaR-diversification-speed is $1-1 / v$. This is above $1 / 2$ if $v>2$ (guaranteeing a finite variance). This intuition is made rigorous below by means of the celebrated Feller convolution theorem for heavy tailed (i.e. regularly varying) distributions.

For the empirical counterpart of this analysis, we briefly review the semiparametric approach to estimating the (extreme) downside risk. The heavy tail feature is captured by a Pareto distribution like term, of which one needs to estimate the tail index (the equivalent of the degrees of freedom $v$ in case of the Student law) and a scale coefficient. We consider estimation by means of a pooled data set on basis of the assumption that the tail indices of the different securities and risk components are equal. We do allow for heterogeneity of the scale coefficients, though. Most securities' distributions display equal hyperbolic tail coefficients, but do differ considerably in terms of their scale coefficients, see Hyung and de Vries (2002). Within this framework it is possible to calculate the diversification effects beyond the sample range and for hypothetically larger portfolios, if we make some assumptions regarding the market model betas and scale coefficients of the orthogonal risk factors. The diversification speeds are analyzed graphically.

We start our essay by reviewing the Feller's convolution theorem for distributions with heavy tails. Subsequently, we study the diversification problem in more detail by adding the market factor. The relevance of the theoretical 
results for the downside risk portfolio diversification question is demonstrated by an application to $\mathrm{S} \& \mathrm{P}$ stock returns.

\section{Diversification Effects and the Feller Convo- lution Theorem}

In this section we only consider securities which are independently distributed. In the next section this counterfactual assumption, as least as far as equities are concerned, is relaxed by allowing for common factors. Let $R_{i}$ denote the logarithmic return of the $i$-th security. Suppose the $\left\{R_{i}\right\}$ are generated by a distribution with heavy tails in the sense of regular variation at infinity. Thus, far from the origin the Pareto term dominates:

$$
\operatorname{Pr}\left\{R_{i} \leq-x\right\}=A_{i} x^{-\alpha}[1+o(1)], \alpha>0, A_{i}>0,
$$

as $x \rightarrow \infty$. The Pareto term implies that only moments up to $\alpha$ are bounded and hence the informal terminology of heavy tails. Per contrast the normal distribution has all moments bounded thanks to the exponential tail shape. Distributions like the Student-t, Pareto, non-normal sum-stable distributions all have regularly varying tails. Downside risk measures like the VaR, i.e. at the desired probability level $\delta: \operatorname{Pr}\left\{R_{i} \leq-\mathrm{VaR}\right\}=\delta$, directly pick up differences in tail behavior.

An implication of the regular variation property is the simplicity of the tail 
probabilities for convoluted data. Suppose the $\left\{R_{i}\right\}$ are generated by a heavytailed distribution which satisfies (1). From the Feller's Theorem (1971, VIII.8), the distribution of the $k$-sum satisfies ${ }^{2}$

$$
\operatorname{Pr}\left\{\sum_{i=1}^{k} R_{i} \leq-x\right\}=k A x^{-\alpha}[1+o(1)], \text { as } x \rightarrow \infty .
$$

From this one can derive the diversification effect for the equally weighted portfolio $\bar{R}=\frac{1}{k} \sum_{i=1}^{k} R_{i}$, see Dacorogna et al. (2001). The following first order approximation for the equally weighted portfolio diversification effect regarding the downside risk obtains ${ }^{3}$

$$
\operatorname{Pr}\left\{\frac{1}{k} \sum_{i=1}^{k} R_{i} \leq-x\right\} \approx k^{1-\alpha} A x^{-\alpha} .
$$

Under the heterogeneity of the scale coefficients $A_{i}$, the equivalent of equation

(2) reads

$$
\operatorname{Pr}\left\{\frac{1}{k} \sum_{i=1}^{k} R_{i} \leq-x\right\} \approx k^{-\alpha}\left(\sum_{i=1}^{k} A_{i}\right) x^{-\alpha} .
$$

To summarize, if at a constant VaR level $x$, one increases the number $k$ of securities included in the portfolio, this decreases the probability of loss by $k^{1-\alpha}$, see (2).

The other case is where the $R_{i}$ are independent standard normally dis-

\footnotetext{
${ }^{2}$ Note that in this analysis $x \rightarrow \infty$, while $k$ is a fixed number.

${ }^{3}$ Note that this diversification result only holds as $x \rightarrow \infty$. Garcia, Renault and Tsafack (2003) show that for symmetric stable distributions, the diversification result applies anywhere below the median. This has to do with the fact that the sum stable distributions are self additive throughout their support, while this only applies in the tail region for the class of fat tailed distributions.
} 
tributed

$$
\operatorname{Pr}\left\{\frac{1}{k} \sum_{i=1}^{k} R_{i} \leq-x\right\} \sim N\left(0, \frac{1}{k}\right)
$$

The following is the equivalent of (1) for the normal distribution

$$
\operatorname{Pr}\left\{R_{i} \leq-x\right\}=\frac{1}{x} \frac{1}{\sqrt{2 \pi}} \exp \left(-\frac{1}{2} x^{2}\right)[1+o(1)] \text { as } x \rightarrow \infty .
$$

For the equally weighted portfolio it thus holds

$$
\operatorname{Pr}\left\{\frac{1}{k} \sum_{i=1}^{k} R_{i} \leq-x\right\}=\operatorname{Pr}\left\{\frac{1}{\sqrt{k}} R_{i} \leq-x\right\} \simeq \frac{1}{x \sqrt{k}} \frac{1}{\sqrt{2 \pi}} \exp \left(-\frac{1}{2} k x^{2}\right)
$$

It follows that under normality

$$
\frac{d \ln \operatorname{Pr}}{d \ln k} \simeq-\frac{1}{2}-\frac{1}{2} x^{2} k
$$

while under fat tail model from equation (2),

$$
\frac{d \ln \operatorname{Pr}}{d \ln k} \simeq 1-\alpha
$$

Hence, for sufficiently high but fixed $k$ the normal distribution implies a higher Diversification-speed-of-the-risk-level.

Next consider holding the probability constant but letting the VaR level change, which is the typical case considered under the safety first criterion, to determine the VaR-diversification-speed. Thus in case of the normal model we 
are interested in comparing VaR levels $t$ and $s$ such that

$$
\operatorname{Pr}\left\{R_{i} \leq-t\right\}=\operatorname{Pr}\left\{\frac{1}{k} \sum_{i=1}^{k} R_{i} \leq-s\right\}=\operatorname{Pr}\left\{\frac{1}{\sqrt{k}} R_{i} \leq-s\right\} . .
$$

Using the additivity properties of the normal distribution, or equivalently using (4) on both sides of (7), it is immediate that

$$
s=\frac{t}{\sqrt{k}} .
$$

So that the normal based VaR-diversification-speed reads

$$
\frac{d \ln s}{d \ln k}=-\frac{1}{2} .
$$

For the fat tailed model the equivalent of $(7)$ is

$$
A_{i} t^{-\alpha}=\operatorname{Pr}\left\{R_{i} \leq-t\right\}=\operatorname{Pr}\left\{\frac{1}{k} \sum_{i=1}^{k} R_{i} \leq-s\right\}=k^{-\alpha}\left(\sum_{i=1}^{k} A_{i}\right) s^{-\alpha} .
$$

Solving for $s$ gives

$$
s=\frac{t}{k}\left(\frac{\sum_{i=1}^{k} A_{i}}{A_{i}}\right)^{1 / \alpha} .
$$

Furthermore, if the scale coefficients are identical this simplifies to

$$
s=\frac{t}{k^{1-1 / \alpha}} .
$$


So that if $\alpha>2$, i.e. when the variance exists,

$$
\frac{d \ln s}{d \ln k}=-\left(1-\frac{1}{\alpha}\right)<-\frac{1}{2} .
$$

Compare ( 9 ) to ( 8 ). If $\alpha>2$, then the VaR-diversification-speed is a higher for fat tailed distributed returns than if the returns were normally distributed.

\section{Diversification Effects in Factor Models}

We relax the assumption of independence between security returns and allow for non-diversifiable market risk. The market risk reduces the benefits from diversification to the elimination of the idiosyncratic component of the risk. First consider a single index model in which all idiosyncratic risk is assumed independent from the market risk $R$

$$
R_{i}=\beta_{i} R+Q_{i}
$$

and where $R$ is the (excess) return on the market portfolio, $\beta_{i}$ is the amount of market risk and $Q_{i}$ is the idiosyncratic risk of the return on asset $i$. The idiosyncratic risk may be diversified away fully in arbitrarily large portfolios and hence is not priced. But the cross-sectional dependence induced by common market risk factor has to be held in any portfolio.

We apply Feller's theorem again for deriving the benefits from cross-sectional portfolio diversification in this single index model. Consider an equally weighted 
portfolio of $k$ assets. Let $\bar{\beta}=\frac{1}{k} \sum_{i=1}^{k} \beta_{i}$. The case of unequally weighted portfolios is but a minor extension left to the reader for consideration of space. In this single index model the $Q_{i}$ are cross-sectionally independent and, moreover, are independent from the market risk factor $R$. Suppose in addition that the $Q_{i}$ satisfy $\operatorname{Pr}\left\{Q_{i} \leq-x\right\} \approx A_{i} x^{-\alpha}$ for all $i$, and that $\operatorname{Pr}\{R \leq-x\} \approx A_{r} x^{-\alpha}$. The diversification benefits from the equally weighted portfolio regarding the downside risk measure for the case of homogenous scale coefficients $A_{i}=A$ then follow as

$$
\operatorname{Pr}\left\{\frac{1}{k} \sum_{i=1}^{k} R_{i} \leq-x\right\} \approx k^{1-\alpha} A x^{-\alpha}[1+o(1)]+\bar{\beta}^{\alpha} A_{r} x^{-\alpha}[1+o(1)]
$$

as $x \rightarrow \infty$. If the scale coefficients are heterogenous, the equivalent of equation (11) reads

$$
\operatorname{Pr}\left\{\frac{1}{k} \sum_{i=1}^{k} R_{i} \leq-x\right\} \approx k^{-\alpha}\left(\sum_{i=1}^{k} A_{i}\right) x^{-\alpha}+\bar{\beta}^{\alpha} A_{r} x^{-\alpha} .
$$

In large portfolios one should see that almost all downside risk is driven by the market factor, if $\alpha>1$

$$
\operatorname{Pr}\left\{\frac{1}{k} \sum_{i=1}^{k} R_{i} \leq-x\right\} \approx \bar{\beta}^{\alpha} A_{r} x^{-\alpha}
$$

for large, but finite $k$.

In general one finds the single index model does not hold exactly due to the fact that $\operatorname{Cov}\left[Q_{i}, Q_{j}\right]$ is typically non-zero for off diagonal elements as well. 
Thus though the $Q_{i}$ may be independent from the market risk factor $R$ (they are uncorrelated with $R$ by construction), they are typically not cross sectionally independent from each other. This case is usually referred to as the market model. For example, let there be one other common factor $F$. This factor is assumed independent from $R$, but the $\operatorname{Cov}\left[Q_{i}, F\right] / \operatorname{Cov}[F, F]=\tau_{i}$ say. Let $\bar{\tau}=\frac{1}{k} \sum_{i=1}^{k} \tau_{i}$, and assume that $\operatorname{Pr}\{F \leq-x\} \approx A_{f} x^{-\alpha}$. Then, by analogy with the foregoing results

$$
\operatorname{Pr}\left\{\frac{1}{k} \sum_{i=1}^{k} R_{i} \leq-x\right\} \approx k^{-\alpha}\left(\sum_{i=1}^{k} A_{i}\right) x^{-\alpha}+\bar{\beta}^{\alpha} A_{r} x^{-\alpha}+\bar{\tau}^{\alpha} A_{f} x^{-\alpha}
$$

To study the case of non-identical $\alpha$ in (12), one has to consider two cases:

Case $1 \alpha_{r}=\alpha_{1}=\ldots=\alpha_{j}<\alpha_{j+1} \leq \alpha_{j+2} \leq \ldots \ldots \leq \alpha_{k}$.

Case $2 \alpha_{1}=\ldots=\alpha_{j}<\alpha_{j+1} \leq \alpha_{j+2} \leq \ldots \ldots \leq \alpha_{k}$ and $\alpha_{r}>\alpha_{1}$.

Here $\alpha_{r}$ stands for the tail index of the market portfolio return, and the $\alpha_{i}$ are the indices of the idiosyncratic parts of the security $i$ return. Then corresponding expressions to (12) are for case (1)

$$
\operatorname{Pr}\left\{\frac{1}{k} \sum_{i=1}^{k} R_{i} \leq-x\right\} \approx k^{-\alpha_{r}}\left(\sum_{i=1}^{j} A_{i}\right) x^{-\alpha_{r}}+\bar{\beta}^{\alpha_{r}} A_{r} x^{-\alpha_{r}}
$$

and for case (2)

$$
\operatorname{Pr}\left\{\frac{1}{k} \sum_{i=1}^{k} R_{i} \leq-x\right\} \approx k^{-\alpha_{1}}\left(\sum_{i=1}^{j} A_{i}\right) x^{-\alpha_{1}}
$$


Next, consider holding the probability constant but letting the VaR level change in (12) as the number of assets $k$ increases. From (12) we had

$$
\operatorname{Pr}\left\{\frac{1}{k} \sum_{i=1}^{k} R_{i} \leq-x\right\} \approx k^{-\alpha}\left[\sum_{i=1}^{k} A_{i}+\left(\sum_{i=1}^{k} \beta_{i}\right)^{\alpha} A_{r}\right] x^{-\alpha}
$$

By first order inversion, cf. De Bruijn's theorem in Bingham et al. (1987), one obtains

$$
V a R=x=\frac{1}{k}\left[\sum_{i=1}^{k} A_{i}+\left(\sum_{i=1}^{k} \beta_{i}\right)^{\alpha} A_{r}\right]^{1 / \alpha} \bar{p}^{-1 / \alpha}
$$

and where $\bar{p}$ is the fixed probability level. With homogenous scale coefficients, we may simplify this to

$$
V a R=\frac{1}{k^{1-1 / \alpha}}\left[A+\frac{\left(\sum_{i=1}^{k} \beta_{i}\right)^{\alpha}}{k} A_{r}\right]^{1 / \alpha} \bar{p}^{-1 / \alpha} .
$$

This should be compared with the results from the previous section on the VaR-diversification-speed, where the part stemming from the market factor was absent. In particular we find

$$
\frac{d \ln V a R}{d \ln k}=-1+\frac{1}{\alpha} \frac{A}{A+\frac{\left(\sum_{i=1}^{k} \beta_{i}\right)^{\alpha}}{k} A_{r}},
$$

which is smaller, i.e. gives a higher speed, than the simple $-1+1 / \alpha$ from before. 


\section{Estimation by Pooling}

To investigate the relevance of the above downside risk diversification theory, we need to estimate the various downside risk components. To explain the details of the estimation procedure, consider again the simple setup in (3). To be able to calculate the downside risk measure, one needs estimates of the tail index $\alpha$ and the scale coefficients $A_{i}$. A popular estimator for the inverse of the tail index is Hill's (1975) estimator. If the only source of heterogeneity are the scale coefficients, one can pool all return series. Let $\left\{R_{11}, \ldots, R_{1 n}, \ldots, R_{k 1}, \ldots, R_{k n}\right\}$ be the sample of returns. Denote by $Z_{(i)}$ the $i$-th descending order statistic from $\left\{R_{11}, \ldots, R_{1 n}, \ldots, R_{k 1}, \ldots, R_{k n}\right\}$. If we estimate the left tail of the distribution, it is understood that we take the losses (reverse signs). The Hill estimator reads

$$
\widehat{1 / \alpha}=\frac{1}{m} \sum_{i=1}^{m} \ln \left(Z_{(i)}\right)-\ln \left(Z_{(m+1)}\right)
$$

This estimator requires a choice of the number of the highest order statistics $m$ to be included, i.e. one needs to locate the start of the tail area. We implemented the subsample bootstrap method proposed by Danielsson et al. (2000) to determine $m$. The estimator for the scale $A$ when $A_{i}=A$ for all $i$ is

$$
\widehat{A}=\frac{m}{k n}\left(Z_{(m+1)}\right)^{\widehat{\alpha}}
$$

Note that $m / n k$ is the empirical probability associated with $Z_{(m+1)}$, and the estimator $\widehat{A}$ follows intuitively from (1). Under the heterogeneity of $A_{i}$ one 
takes

$$
\widehat{A}_{i}=\frac{m_{i}}{n}\left(Z_{(m+1)}\right)^{\widehat{\alpha}}
$$

where $m_{i}$ is such that

$$
R_{i(1)} \geq \ldots \geq R_{i\left(m_{i}\right)} \geq Z_{(m+1)} \geq R_{i\left(m_{i}+1\right)} \geq \ldots \geq R_{i n}
$$

Note that $\sum_{i=1}^{k} m_{i}=m$. This implies that by the pooling method we obtain exactly the same portfolio probabilities whether or not one assumes (counterfactually incorrect) identical or heterogenous scale coefficients, since

$$
\begin{aligned}
k^{-\widehat{\alpha}}\left(\sum_{i=1}^{k} \widehat{A}_{i}\right) x^{-\widehat{\alpha}} & =k^{-\widehat{\alpha}}\left(\sum_{i=1}^{k} \frac{m_{i}}{n}\left(Z_{(m+1)}\right)^{\widehat{\alpha}}\right) x^{-\widehat{\alpha}} \\
& =k^{-\widehat{\alpha}} \frac{\left(\sum_{i=1}^{k} m_{i}\right)}{n}\left(Z_{(m+1)}\right)^{\widehat{\alpha}} x^{-\widehat{\alpha}} \\
& =k^{1-\widehat{\alpha}} \widehat{A} x^{-\widehat{\alpha}}
\end{aligned}
$$

We can adapt this pooling method to the market model with little modification. Pooling the series $\{R\},\left\{Q_{1}\right\}, \ldots\left\{Q_{k}\right\}$, one can use the same procedure as in the case of cross-independence. ${ }^{4}$ For the estimation of the tail index one uses again (15), where in this case $\{Z\}=\left\{R_{r 1}, \ldots, R_{r n}, Q_{11}, \ldots, Q_{1 n}, \ldots, Q_{k 1}, \ldots, Q_{k n}\right\}$

\footnotetext{
${ }^{4}$ The determination of the parameters $\beta_{i}$ and the residuals $Q_{i}$ entering in the definition of the market model is done by regressing the stock returns on the market return. The coefficient $\beta_{i}$ is thus given by the ordinary least squares estimator, which is consistent as long as the residuals are white noise and have zero mean and finite variance. The idiosyncratic noise $Q_{i}$ is obtained by subtracting $\beta_{i}$ times the market return to the stock return.
} 
Estimators for the scales are

$$
\widehat{A}_{i}=\frac{m_{i}}{n}\left(Z_{(m+1)}\right)^{\widehat{\alpha}}, i=1, \ldots, k \text { and } r,
$$

where $m_{i}$ is such that

$$
X_{i(1)} \geq \ldots \geq X_{i\left(m_{i}\right)} \geq Z_{(m+1)} \geq X_{i\left(m_{i}+1\right)} \geq \ldots \geq X_{i n},
$$

where $X_{i}$ can be $R$ or $Q_{i}$.

In case the tail indices differ across securities and risk factors, the above can be easily adapted to estimation on individual series. There is however considerable evidence that the tail indices are comparable for equities from the S\&P 500 index, see e.g. Jansen and De Vries (1991) and Hyung and De Vries (2002). Therefore we decided to proceed on basis of the assumption that the tail indices are equal.

\section{Empirical Analysis of the Diversification Speed}

We now apply our theoretical results to the daily returns of a set of stocks. In order to estimate the parameters of the market model we choose the Standard and Poor's 500 index as a representation of the market factor. This is certainly not the market portfolio as in the CAPM; nevertheless, the S\&P 500 index represents about $80 \%$ of the total market capitalization. To see the effects of portfolio diversification, we choose 15 stocks arbitrarily from the S\&P 100 index 
in March of 2001. We use the daily returns (close-to-close data), including cash dividends. The data were obtained from the Datastream. The data span runs from January 2, 1980, through March 6, 2001, giving a sample size of $n=5,526$. Thus more than 20 years of daily data are considered, including the short-lived 1987 crash. All results are in terms of the excess returns above the risk free interest rate (three month US Treasury bills).

The summary statistics for each stock return series and the market factor are given in Table 1. On an annual basis the excess returns hover around $7.5 \%$ and have comparable second moments. The excess returns all exhibit considerably higher than normal kurtosis. This latter feature is also captured by the estimates of the tail index $\alpha$ in Table 2 . In this table we report tail index and scale estimates using the individual series, counter to the pooling method outlined above. This is done in order to show that the tail indices are indeed rather similar, while there is considerable variation in the scales. This motivates the single tail index, heterogenous scale model implemented in the other tables. Table 2 also gives the beta estimates for the market model.

In Table 3 computations proceed by using the pooling method, assuming identical tail indices for all risk components. We report the estimates of the scale parameter $A$, and the optimal number of order statistics $m$. Both are calculated for the series of excess returns and for the (constructed) orthogonal residuals from the market model (using the betas). The tail index estimate using all excess returns is 3.163 , while when we use all the residuals the tail index is 3.246. The scale parameter estimates, however, differ considerably since these 
range between 14.4 and 46.4 for the excess returns, and are between 4.3 and 42.2 for the market returns and residuals respectively. We note that the scale estimates for the excess returns using the pooling method are more homogeneous than when using the individual series approach from Table 2.

The effects of portfolio diversification are reported in Table 4. The downside risk measure is the probability of a loss in excess of the VaR level $s$; we report at four different loss levels (respectively $s=7.10,11.69,13.33$ and 15.97) $)^{5}$. Four different levels of portfolio aggregation are considered: one stock, 5 stocks, 10 stocks and 15 stocks. The numbers in row EMP are the probabilities from the empirical distribution function of the total return series. The normal law is often used as the workhorse distribution model in finance, even though it does not capture the characteristic tail feature of the data. Therefore in the rows labelled $N O R$ we give the probabilities from the normal model based formula, using the mean and variance estimates from the averaged series. The estimated values in rows $F A T$ were obtained by the heavy tail model using the averaged total excess returns $\sum_{i=1}^{k} R_{i} / k$. The rows $C D p$ give the probability estimates from the pooled series on the basis of (12) assuming the heterogenous scale model. One notes that the normal model does well in the center, but performs poorly as one moves into the tail part. Per contrast, the averaged series in rows FAT is always quite close to the empirical distribution function in the tail area. This shows that the heavy tail model much better captures the tail properties. If we turn to the last rows, one notes that the model in (12) does

\footnotetext{
${ }^{5}$ We choose these particular set of VaR values from the 5.0, 1.0, 0.5 and $0.25 \%$ quantiles of the market returns.
} 


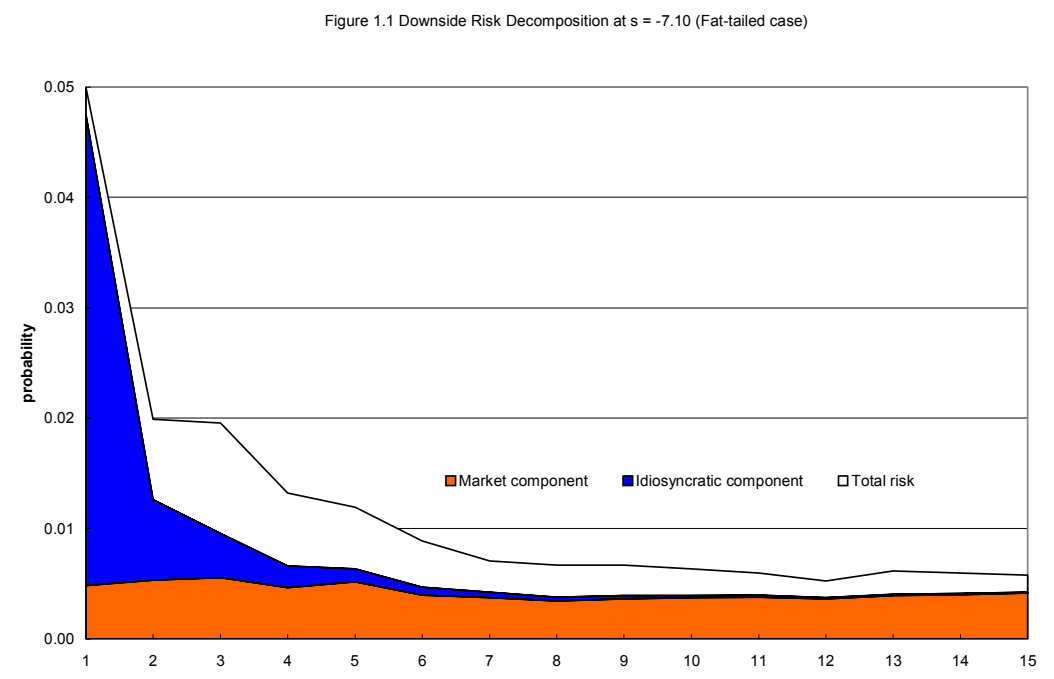

capture a considerable part of the tail risk of the portfolio, but that there is a gap between the tail risk which is explained by the model and which is left unexplained. This is further interpreted below.

To judge these results and to study the speed of diversification a graphical exposition is insightful. In Figures 1.1 and 1.2 we show the Diversification-speedof-the-risk-level by plotting the probability of loss for two different VaR levels against the number of securities which are included in the portfolio ${ }^{6}$. Figure 1.1 is for the 7.10 VaR level, and Figure 1.2 concerns the 15.97 VaR level. The top line gives the total amount of tail risk by means of the empirical distribution function. The grey area constitutes the market risk component, while the black area contains the idiosyncratic risk from (12). Note that the idiosyncratic risk is basically eliminated once the portfolio includes about seven stocks. To put this

${ }^{6}$ The order by which the securities are included corresponds to the numbering in Table 1. 
Figure 1.2 Downside Risk Decomposition at $\mathrm{s}=-15.97$ (Fat-tailed case)

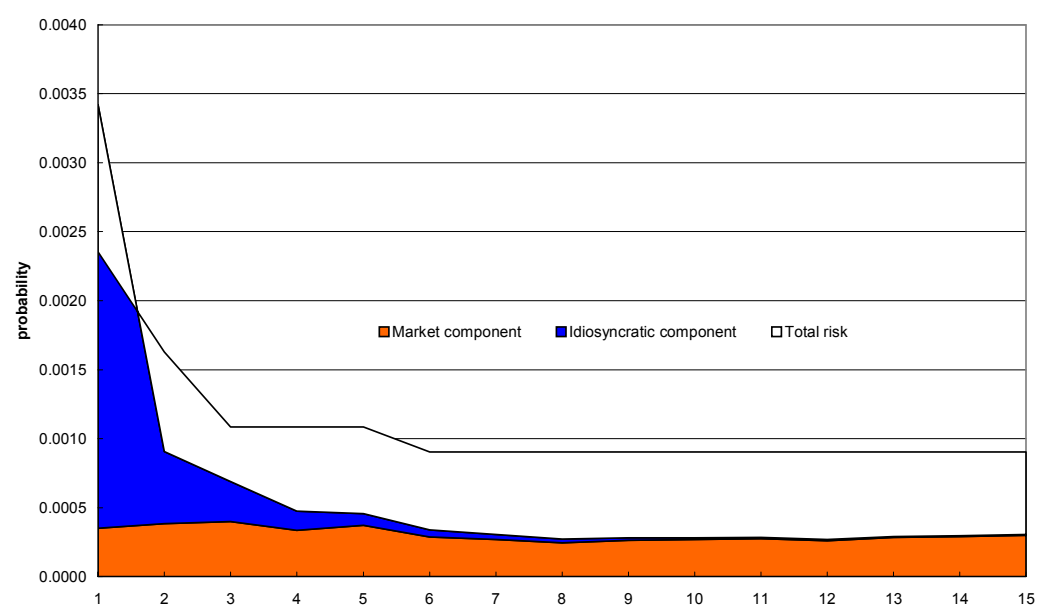

Figure 2. Variance Decomposition

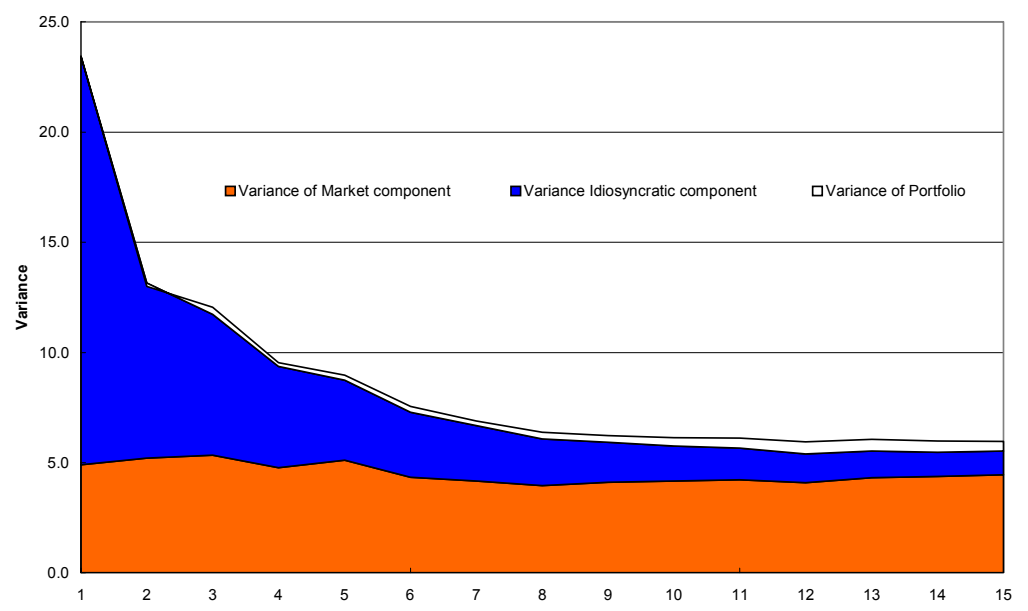


result into perspective, we also provide a graph for the speed of diversification concerning the variance, see Figure 2. This is a global risk measure and under independence, the variance of the idiosyncratic part should decline linearly in $k$. As can be seen from this latter figure, it takes approximately double the number of securities to eliminate the variance part contributed by the idiosyncratic risk part, cf. Elton and Gruber (1995). Note that this corroborates the rate given in (6) and the value of $\alpha \simeq 3$ as in Table 2 (while the variance declines at speed 1). Interestingly as noted at the end of the previous paragraph, another remarkable difference between the last figure and the first two figures is the size of the residual risk driven by the factors other than the market factor. While this component is relatively minor for the variance risk measure, it is even larger than the market risk component for the downside risk measure. This points to the presence of another factor $F$ uncorrelated with $R$ as in (13). This other factor induces a small correlation between the residuals, see Figure 2. This small correlation not withstanding, the other factor appears important with respect to the downside risk. In future research we hope to relate this factor to economic variables.

Next we compare the VaR-diversification-speed under the normal model with the fat tail model. To plot the VaR-diversification-speed we now look in the VaR- $k$ space. From (14) it is clear one cannot separate the market part form the idiosyncratic part, due to the power $1 / \alpha$. Nevertheless, one can first plot the VaR level doing as if only the market factor were relevant (e.g. this would be the case if the idiosyncratic risks have a higher tail index compared to the 
market index). The market factor is from (14)

$$
x=\left(\frac{1}{k} \sum_{i=1}^{k} \beta_{i}\right)\left[A_{r}\right]^{1 / \alpha} \bar{p}^{-1 / \alpha} .
$$

The next line plots the combined effect, market factor and idiosyncratic components, which simply is (14). Third, one plots the empirical quantile function as more assets are added. Similarly, one can proceed in this fashion under the assumption that the returns follow the normal distribution.

Figure 3.1 - Figure 4.2 show the decreasing level of VaR for the given probability. Figure 3.1 is for the 0.05 probability level, and Figure 3.2 concerns the 0.0025 probability level in case of the fat tailed distribution. The top line gives the total amount of VaR by means of the empirical distribution function. The grey area constitutes the VaR level from market risk component as in (16), while the black area plus the grey area displays (14). Figure 4.1 is for the 0.05 probability level, and Figure 4.2 concerns the 0.0025 probability level for the case of the normal distribution. These figures clearly display the theoretical prediction (9), that the VaR-diversification-speed for the idiosyncratic risk is lower for the normal model than for the fat-tailed model.

\section{Out-of-sample, Out-of-portfolio}

The semi-parametric approach we followed to construct the downside risk measure can also be used to go beyond the sample. We consider two possible applications of this technique which might be of use to risk managers. The first 
Figure 3.1 VaR Decomposition at $p=0.05$ (Fat-tailed case)

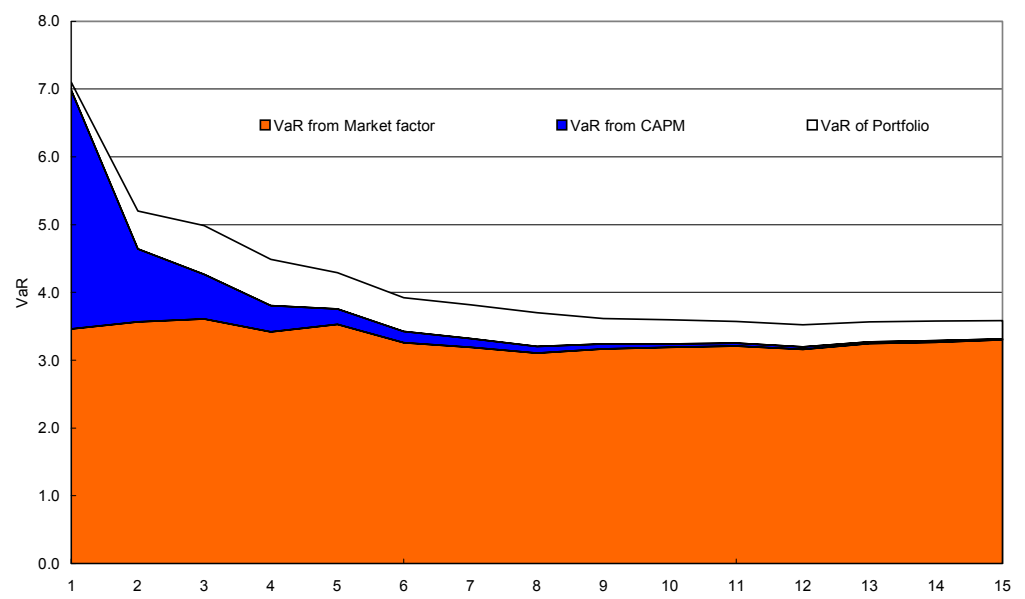

Figure 32 VaR Decomposition at $\mathrm{p}=0.0025$ (Fat-tailed case)

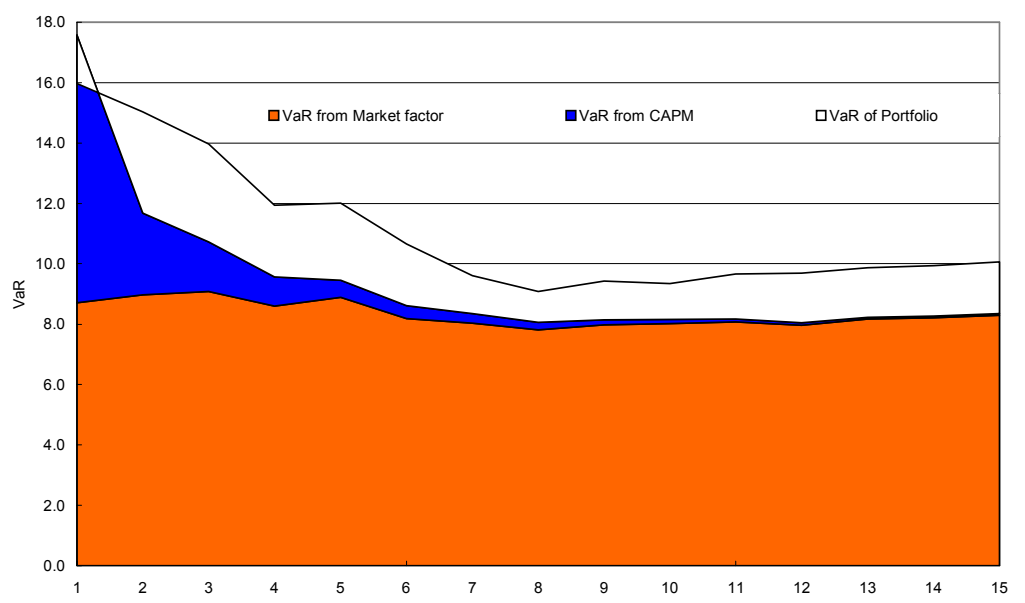


Figure 4.1 VaR Decomposition at $p=0.05$ (Normal case)

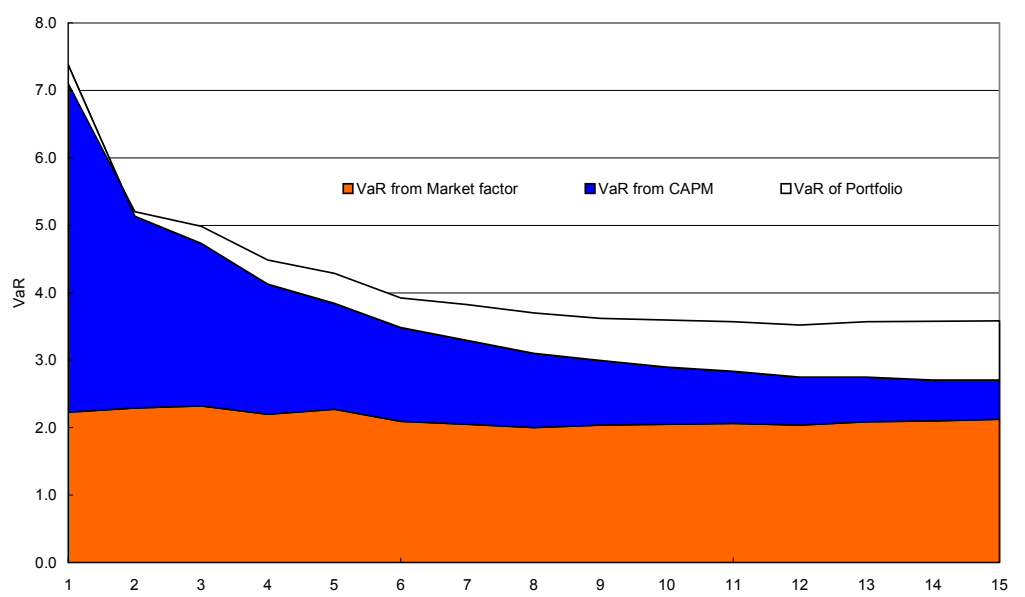

Figure 4.2 VaR Decomposition at $p=0.0025$ (Normal case)

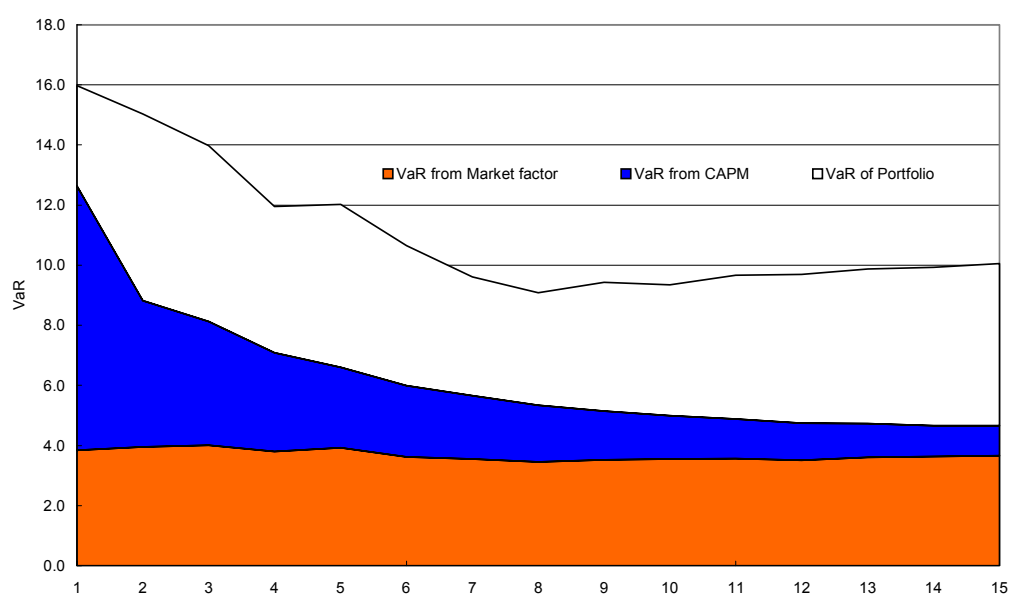


application asks the question how much extra diversification benefits could be derived from adding more securities, without having observations on these securities. By making an assumption regarding the value of the average beta and the average scale of the residual risk factors in the enlarged portfolio, one can use (12) to extrapolate to larger than sample size portfolios. A second application is to increase the loss levels at which one wants to evaluate the downside risk level beyond the worst case in sample. Moreover, even at the border of the sample our approach has real benefits. By its very nature the empirical distribution is bounded by the worst case and hence has its limitations, since the worst case is a bad estimator of the quantile at the $1 / n$ probability level (and vice versa). Thus increasing the loss level $x$ in (12) beyond the worst case gives an idea about the risk of observing even higher losses.

In Table 5 the block denoted as Case I just summarizes some information from the previous Table 4. The Case III block addresses the first application by increasing the number of securities $k$ beyond the sample value of 15 . We assumed the following average beta values: $\bar{\beta}=0.7,0.83$ and 0.9 . The Case II block increases the loss return level. In Table 4 we used 15.97 as the highest loss level. Above this level many securities have no observations. There is one equity with much higher loss returns and we used this one to provide the 'out of sample' loss levels of 22.03, 25.21, 33.69 and 40.45 respectively. To interpret Case III, note that the inclusion of more stocks that have a close correlation with the market component increases the loss probability for a given VaR level. For example consider a portfolio of $k=30$ stocks, at the -15.97 quantile when 
$\bar{\beta}=0.7$ the probability is 0.0169 but when $\bar{\beta}=0.9$ the probability increases to 0.0381 .

\section{Conclusion}

Risk managers use portfolios to diversify away the un-priced risk of individual securities. In this paper we study the benefits of portfolio diversification with respect to extreme downside risk, or the VaR risk measure. The risk of a security is decomposed into a part which is attributable to the market risk and an independent risk factor. The independent part consists of an idiosyncratic part and a second common factor. Two different measures for diversification effects are studied. The VaR-diversification-speed measure holds the probability level constant and gives the rate of change by which the VaR declines as more securities are added to the portfolio, while the Diversification-speed-of-the-risk-level holds the VaR level constant and measures the decline in the probability level. For the VaR-diversification-speed measure we argued fat tailed distributed idiosyncratic risk factors should go down at a higher speed than normal distributed idiosyncratic risk factors. This theoretical prediction was also found empirically to be the case. Furthermore, we provide predictions for the downside risk diversification benefits beyond the range of the empirical distribution function.

This research can be extended in several directions. Given the large gaps in Figures 1 and 2 between the total downside risk and the market factor downside risk contribution, it is of interest to see whether one can identify the remaining 
risk factors $F$ as in (13). Moreover, one would like to explain why these remaining risk factors are relatively unimportant for the global risk measure such as the variance. Moreover, the above analysis may explain why many investors seem to hold not so well diversified portfolios if a global risk measure like the variance is used as the yardstick.

\section{References}

Arzac, E., and V. Bawa. (1977). "Portfolio choice and equilibrium in capital markets with safety first investors." Journal of Financial Economics 4, 277288.

Bingham, N.H., C.M. Goldie, and J.L. Teugels. (1987). Regular Variation, Cambridge University Press, Cambridge.

Dacorogna, M.M., U.A. Müller, O.V. Pictet, and C.G. de Vries. (2001). "Extremal forex returns in extremely large data sets." Extremes 4, 105-127.

Danielsson, J., L. de Haan, L. Peng, and C.G. de Vries. (2000). "Using a bootstrap method to choose the sample fraction in tail index estimation." Journal of Multivariate Analysis 76, 226-248.

Elton, E.J., and M.J. Gruber. (1995). Modern Portfolio Theory and Investment Analysis 5th ed., Wiley, New York.

Feller, W. (1971). An Introduction to Probability Theory and Its Applications Vol. II, Wiley, New York. 
Garcia, R., E. Renault, and G. Tsafack. (2003). "Proper conditioning for coherent VaR in portfolio management." paper presented at the CFS workshop November 2003.

Gourieroux, C., J.P. Laurent, and O. Scaillet. (2000). "Sensitivity analysis of values at risk." Journal of Empirical Finance 7, 225-246.

Hill, B.M. (1975). "A simple general approach to inference about the tail of a distribution." Annals of Statistics 3, 1163-1173.

Hyung, N. and C.G. de Vries. (2002). "Portfolio diversification effects and regular variation in financial data." Allgemeines Statistisches Archiv / Journal of the German Statistical Society 86, 69-82.

Jansen, D., and C.G. de Vries. (1991). "On the frequency of large stock returns: Putting booms and busts into perspective." Review of Economics and Statistics $73,18-24$

Jansen, D., K.G. Koedijk, and C.G. de Vries. (2000). "Portfolio selection with limited downside risk." Journal of Empirical Finance 7, 247-269. 
Table 1. Selected Stocks and Summary Statistics of Excess returns

\begin{tabular}{clccrr}
\hline Series & \multicolumn{1}{c}{ Name } & $\mu_{1}$ & $\mu_{2}$ & \multicolumn{1}{c}{$\mu_{3}$} & \multicolumn{1}{c}{$\mu_{4}$} \\
\hline$m$ & S\&P 500 Index & .0747 & 2.52 & -2.31 & 55.49 \\
1 & ALCOA & .0707 & 4.84 & -0.26 & 13.39 \\
2 & AT \& T & .0392 & 4.33 & -0.35 & 16.41 \\
3 & BLACK \& DECKER & -.0168 & 5.61 & -0.32 & 10.57 \\
4 & CAMPBELL SOUP & .0897 & 4.37 & 0.28 & 9.06 \\
5 & DISNEY (WALT) & .0981 & 4.86 & -1.30 & 29.82 \\
6 & ENTERGY & .0454 & 4.06 & -0.97 & 23.66 \\
7 & GEN.DYNAMICS & .0764 & 4.53 & 0.26 & 10.24 \\
8 & HEINZ HJ & .0968 & 3.99 & 0.11 & 6.35 \\
9 & JOHNSON \& JOHNSON & .1053 & 4.08 & -0.32 & 9.45 \\
10 & MERCK & .1212 & 3.96 & -0.03 & 6.31 \\
11 & PEPSICO & .1170 & 4.43 & -0.04 & 7.82 \\
12 & RALSTON PURINA & .1077 & 4.08 & 0.70 & 15.41 \\
13 & SEARS ROEBUCK & .0542 & 4.91 & -0.24 & 16.83 \\
14 & UNITED TECHNOLOGIES & .0851 & 4.19 & -0.10 & 6.83 \\
15 & XEROX & -.0423 & 5.48 & -1.78 & 33.74
\end{tabular}

Note: Observations cover 01/01/1980 - 03/06/2001, giving 5526 daily observations. The $\mu_{1}, \mu_{2}, \mu_{3}$ and $\mu_{4}$ denote the sample mean, standard error, skewness and kurtosis of annualized excess returns, respectively. The estimates are reported in terms of the excess returns above the risk free interest rate (US Treasury bill 3 months). 
Table 2. Left Tail Parameter Estimates

\begin{tabular}{ccrr}
\hline \hline Series & $\alpha$ & \multicolumn{1}{c}{$A$} & \multicolumn{1}{c}{$m$} \\
\hline$R_{m}$ & 2.963 & 2.522 & 298 \\
1 & 3.789 & 110.117 & 113 \\
2 & 2.785 & 7.953 & 289 \\
3 & 3.220 & 58.601 & 136 \\
4 & 3.505 & 48.766 & 68 \\
5 & 2.549 & 6.211 & 496 \\
6 & 1.981 & 1.339 & 682 \\
7 & 3.218 & 27.687 & 140 \\
8 & 3.404 & 25.811 & 197 \\
9 & 3.377 & 23.663 & 292 \\
10 & 4.035 & 104.724 & 62 \\
11 & 3.789 & 103.171 & 71 \\
12 & 3.136 & 14.106 & 190 \\
13 & 3.166 & 28.244 & 256 \\
14 & 4.335 & 288.036 & 66 \\
15 & 2.098 & 2.999 & 537 \\
\hline \hline
\end{tabular}

$\overline{\text { Note: The values in columns } \alpha,} A$, and $m$ are respectively the tail index, the scale parameter, the estimated optimal number of order statistics and market model beta. 
Table 3. Left Tail Parameter Estimates

\begin{tabular}{|c|c|c|c|c|c|}
\hline \multirow[b]{2}{*}{ Series } & \multicolumn{2}{|c|}{ Excess returns } & \multirow[b]{2}{*}{$\beta$} & \multicolumn{2}{|c|}{ "Residuals } \\
\hline & $A$ & $m$ & & $A$ & $m$ \\
\hline$T$ & 23.0 & 1609 & - & 19.6 & 1021 \\
\hline$R_{m}$ & - & - & 1 & 4.3 & 15 \\
\hline 1 & 26.2 & 122 & 0.877 & 24.7 & 86 \\
\hline 2 & 19.5 & 91 & 0.929 & 15.2 & 53 \\
\hline 3 & 46.4 & 216 & 0.938 & 42.2 & 147 \\
\hline 4 & 22.7 & 106 & 0.719 & 19.5 & 68 \\
\hline 5 & 24.0 & 112 & 1.012 & 22.1 & 77 \\
\hline 6 & 14.4 & 67 & 0.475 & 14.9 & 52 \\
\hline 7 & 25.3 & 118 & 0.710 & 25.0 & 87 \\
\hline 8 & 16.3 & 76 & 0.640 & 14.9 & 52 \\
\hline 9 & 13.9 & 65 & 0.927 & 10.6 & 37 \\
\hline 10 & 15.7 & 73 & 0.854 & 11.5 & 40 \\
\hline 11 & 24.2 & 113 & 0.867 & 18.7 & 65 \\
\hline 12 & 15.0 & 70 & 0.669 & 16.4 & 57 \\
\hline 13 & 29.0 & 135 & 1.074 & 17.5 & 61 \\
\hline 14 & 20.2 & 94 & 0.895 & 13.2 & 46 \\
\hline 15 & 32.4 & 151 & 0.949 & 26.7 & 93 \\
\hline
\end{tabular}

Note: The values in row $T$ give estimates from the pooled series imposing scale homogeneity. The values in rows $R_{m}, 1,2, \ldots, 15$ give estimates for the market returns and the individual stock series for the total excess returns and the residual parts. The values in columns $A$ and $m$ are the scale parameter and the estimated optimal number of order statistics imposing identical tail indices. The values in column $\beta$ are the market model beta. 
Table 4. Lower Tail Probabilities in Percentages

\begin{tabular}{|c|c|c|c|c|c|c|c|c|}
\hline \multirow{2}{*}{$\begin{array}{l}s \\
k\end{array}$} & \multicolumn{4}{|c|}{-7.10} & \multicolumn{4}{|c|}{$\overline{-11.69}$} \\
\hline & 1 & 5 & 10 & 15 & 1 & 5 & 10 & 15 \\
\hline$E M P$ & 4.995 & 1.195 & 0.633 & 0.579 & 0.995 & 0.253 & 0.145 & 0.145 \\
\hline NOR & 7.325 & 0.934 & 0.225 & 0.198 & 0.817 & 0.005 & 0.000 & 0.000 \\
\hline$F A T$ & 6.551 & 1.181 & 0.741 & 0.706 & 0.988 & 0.265 & 0.185 & 0.171 \\
\hline$C D p$ & - & 0.633 & 0.392 & 0.423 & - & 0.125 & 0.078 & 0.084 \\
\hline$s$ & \multicolumn{4}{|c|}{-13.33} & \multicolumn{4}{|c|}{$-15.9^{7}$} \\
\hline$k$ & 1 & 5 & 10 & 15 & 1 & 5 & 10 & 15 \\
\hline$E M P$ & 0.489 & 0.163 & 0.109 & 0.127 & 0.235 & 0.109 & 0.090 & 0.090 \\
\hline NOR & 0.309 & 0.000 & 0.000 & 0.000 & 0.051 & 0.000 & 0.000 & 0.000 \\
\hline$F A T$ & 0.603 & 0.179 & 0.129 & 0.118 & 0.304 & 0.104 & 0.078 & 0.071 \\
\hline$C D p$ & - & 0.082 & 0.051 & 0.055 & - & 0.046 & 0.028 & 0.030 \\
\hline
\end{tabular}

Note: The entries in rows EMP are the probabilities from the empirical distribution. The rows NOR and FAT report the probabilities calculated directly from the parameters of the averaged series itself, where in the former case one uses the presumption of normality and in the latter case regular variation is imposed. The numbers in rows $C D p$ are the probabilities estimated using the pooled series. The $k$ denotes the number of individual stocks included in the averaged series, and $s$ is the loss quantile. Note probabilities are written in percentage format. 
Table 5. Lower Tail Probabilities: Beyond the Sample and the Market

\begin{tabular}{|c|c|c|c|c|c|c|c|c|c|}
\hline \multirow[b]{3}{*}{$k$} & $s$ & -7.10 & -11.69 & -13.33 & -15.97 & -22.03 & -25.21 & -33.69 & -40.45 \\
\hline & $\%$ & 5.0 & 1.0 & 0.5 & 0.25 & 0.090 & 0.054 & 0.018 & 0.009 \\
\hline & & \multicolumn{4}{|c|}{ CASE I } & \multicolumn{4}{|c|}{ CASE II } \\
\hline \multirow{3}{*}{5} & $E M P$ & 1.1946 & .2534 & .1629 & .1086 & .0362 & .0362 & .0181 & .0181 \\
\hline & $F A T$ & 1.1900 & .2660 & .1798 & .1045 & .0397 & .0265 & .0111 & .0064 \\
\hline & $C D p$ & .6093 & .1205 & .0789 & .0439 & .0154 & .0100 & .0039 & .0021 \\
\hline \multirow{3}{*}{10} & $E M P$ & .6335 & .1448 & .1086 & .0905 & .0181 & .0181 & .0181 & .0181 \\
\hline & $F A T$ & .6800 & .1490 & .1001 & .0578 & .0217 & .0144 & .0060 & .0034 \\
\hline & $C D p$ & .3914 & .0774 & .0507 & .0282 & .0099 & .0064 & .0025 & .0014 \\
\hline \multirow{3}{*}{15} & $E M P$ & .5792 & .1448 & .1267 & .0905 & .0181 & .0181 & .0181 & .0181 \\
\hline & $F A T$ & .7087 & .1722 & .1189 & .0712 & .0286 & .0195 & .0086 & .0051 \\
\hline & $C D p$ & .4227 & .0836 & .0547 & .0304 & .0107 & .0069 & .0027 & .0015 \\
\hline \multicolumn{10}{|c|}{ CASE III } \\
\hline \multirow{3}{*}{20} & $C D p 1$ & .2375 & .0470 & .0307 & .0171 & & & & \\
\hline & $C D p^{2}$ & .4190 & .0829 & .0543 & .0302 & & & & \\
\hline & $C D p 3$ & .5318 & .1052 & .0689 & .0383 & & & & \\
\hline \multirow{3}{*}{25} & $C D p 1$ & .2359 & .0467 & .0305 & .0170 & & & & \\
\hline & $C D p^{2}$ & .4175 & .0826 & .0541 & .0301 & & & & \\
\hline & $C D p 3$ & .5302 & .1049 & .0687 & .0382 & & & & \\
\hline \multirow{3}{*}{30} & $C D p 1$ & .2350 & .0465 & .0304 & .0169 & & & & \\
\hline & $C D p^{2}$ & .4166 & .0824 & .0540 & .0300 & & & & \\
\hline & $C D p 3$ & .5294 & .1047 & .0686 & .0381 & & & & \\
\hline
\end{tabular}

Note: The entries in rows EMP are the probabilities from the empirical distribution. The numbers in rows FAT are the probabilities calculated directly from the parameters of averaged series itself. The numbers in row $C D p$ are the probabilities from the fat tail market model (12). The numbers in rows $C D p 1$,2and 3 are calculated by imposing $\bar{\beta}=0.7,0.8358$ and 0.9 , respectively. The $k$ denotes the number of individual stocks included in the averaged series, and $s$ gives the loss quantile. Note probabilities are written in percentage format. 